\title{
Relativas y Anteposición Negativa en inglés y español ${ }^{\mathbf{1}}$
}

\author{
Relative clauses and Negative Preposing in English and Spanish
}

Ángel Luis Jiménez Fernández ${ }^{1}$

1Universidad de Sevilla

Recibido: 17/06/2020; Aceptado: 28/09/2020

\section{Resumen}

En este trabajo analizo el impacto de los denominados Fenómenos de Oración Matriz, es decir, operaciones de índole discursiva que se dan exclusivamente en oraciones principales, como la Anteposición Negativa (ANeg), en oraciones relativas especificativas y explicativas, estableciendo un contraste entre el inglés y el español desde una perspectiva minimista (p. ej. This car, which only rarely did I drive, is in excellent condition). A tal efecto, he realizado un experimento con nativos de ambas lenguas, donde la tarea era juzgar la aceptabilidad de los tipos de relativas que incluían ANeg. El resultado más intrigante consiste en que en español no hay distinción entre los tipos de relativas que aceptan la ANeg, mientras que en inglés solo la permiten las relativas explicativas. La variación detectada en este trabajo experimental se explica mediante un análisis formal basado en el mecanismo de herencia de rasgos discursivos y la diferencia entre aserción y presuposición, que se refleja en la derivación sintáctica mediante la inclusión de un operador factivo (noasertivo) que bloqueará o no la ANeg, dependiendo de si esta tiene que cruzar el operador no-asertivo en su movimiento a la periferia izquierda de la oración en una lengua determinada.

Palabras clave: relativas especificativas y explicativas, Anteposición Negativa, experimento, contraste inglés/español, aserción vs. presuposición

\section{Abstract}

In this article I explore the impact of so-called Main Clause Phenoma, that is discourse-driven operations which occur mainly in main or root clauses, such as Negative Preposing (NPr) in restrictive and non-restrictive relative clauses, making a contrast between English and Spanish from a minimalist perspective (e.g. This car, which only rarely did I drive, is in excellent condition). To this effect, I have carried out an experiment with native speakers of English and Spanish, respectively, where the main task was to judge the acceptability of the two types of relatives when combining with NPr. The most puzzling effect was that Spanish accepted NPr in both types whereas English only allowed for NPr in non-restrictive relatives. I account for this experimental variation by proposing a formal analysis based on the strategy of inheritance of discourse features and the distinction between assertion and presupposition, which is reflected by including a non-assertive operator in the syntactic derivation. This operator does or does not block NPr depending on whether the latter crosses the former in its movement to the left periphery of the sentence in a given language.

Keywords: restrictive and non-restrictive relatives, Negative Preposing, experimental work, English/Spanish contrast, assertion vs. presupposition 


\section{INTRODUCCIÓN}

La Anteposición Negativa (ANeg) consiste en mover a la periferia oracional izquierda un constituyente con propiedades de foco y negación, como en (1) para el inglés y (2) para el español:

(1) No other colleague would he turn to. (Radford 2009: 329)

'A ningún compañero recurriría yo'.

(2) De ninguno de esos problemas trató la reunión. (Bosque 1980: 25)

Está generalmente aceptado que la distinción entre aserción y presuposición es crucial en la legitimación de Fenómenos de Oración Matriz (FOM), que típicamente aparecen en oraciones principales y en algunas subordinadas (Hooper y Thompson 1973, Haegeman 2012, JiménezFernández y Miyagawa 2014, Jiménez-Fernández 2018 y 2020, Miyagawa 2017, De Cat 2012, Heycock 2006).

Las oraciones matrices y pseudomatrices (aquellas subordinadas con propiedades de oraciones principales) permiten los FOMs porque expresan algún tipo de aserción. Así, podemos encontrar ejemplos como los de (3) y (4), donde la subordinada seleccionada por un determiando verbo es compatible con la presencia de FOMs como la topicalización en inglés o la anteposición resuntiva en español (Jiménez-Fernández 2020):

(3) We saw that each part he had examined carefully. (Hooper y Thompson 1973: 125)

'Vimos que cada parte la había examinado él cuidadosamente'.

(4) Juan dijo que el mismo vestido se había comprado Marta.

Esta compatibilidad de los FOMs en oraciones subordinadas viene determinada por la naturaleza asertiva de estas. Es decir, estas subordinadas - al igual que las oraciones principalesson aserciones, y por tanto expresan información que aún no se ha añadido al denominado Fondo Común o Common Ground (Potts 2007: 666). Es precisamente por expresar esta información nueva por lo que los FOMs pueden aparecer en estas subordinadas.

Sin embargo, en otras subordinadas donde la información ya forme parte del Fondo Común y, por tanto, esta constituya información presupuesta, estos FOMs no son aceptables²:

(5) *It was impossible that each part he had examined carefully. (Hooper y Thompson 1973: 109)

'Era imposible que cada parte la hubiera examinado él cuidadosamente'. 
(6) *Me disgusta que la misma propuesta hiciera también el partido de gobierno. (Jiménez-Fernández y Miyagawa 2014: 297)

El estatuto de FOM de la ANeg ha sido ampliamente reconocido por lingüistas como Emonds $(1969,2004)$, Haegeman (2012) y Heycock (2006), entre muchos otros. De este modo, podemos encontrar ejemplos de subordinadas del inglés que rechazan la ANeg. Obsérvese el contraste entre (7) y (8):

(7) I found out that never before had he had to borrow money. (Hooper y Thompson 1973: 480, ej. 119)

'Descubrí que nunca antes había tenido que pedir él dinero prestado'.

(8) *He was surprised that never in my life had I seen a hippopotamus. (Hooper y Thompson 1973: 479, ej. 103)

'Se sorprendió de que nunca en la vida hubiera visto yo un hipopótamo'.

Mientras que la subordinada en (7) expresa aserción, la de (8) indica presuposición. Esta propiedad sirve de característica definitoria para distinguir entre subordinadas cuasimatrices y verdaderas subordinadas. La consecuencia inmediata es que solo las cuasimatrices van a ser compatibles con la ANeg. Nótese que las versiones españolas de (7) y (8) son totalmente gramaticales y aceptables, sin que haya ninguna diferencia entre subordinadas asertivas $y$ presupuestas en cuanto a la legitimación de la ANeg. Esta diferencia entre el español y el inglés en cuanto a la legitimación de la ANeg en contextos subordinados motiva la pregunta de si en otros tipos de subordinadas se detecta dicha distinción.

Las oraciones relativas también han sido objeto de estudio según si son asertivas (relativas explicativas) o presupuestas (relativas especificativas). De aquí podemos inferir que en las especificativas no se van a aceptar los FOMs, mientras que estos serán totalmente lícitos en las relativas explicativas, ya que estas últimas son asertivas, y se agrupan con las oraciones matrices en este sentido. Este distinto comportamiento queda ilustrado en (9a-b), tomados de Hooper y Thompson (1973: 489, ejs. 198 y 199):

(9) a. *The car that only rarely did I drive is in excellent condition.

b. This car, which only rarely did I drive, is in excellent condition.

Sin embargo, los juicios de estas construcciones son muy poco sólidos pues otros autores consideran oraciones como (9a) totalmente aceptables (Culicover, 1991, Radford, 2019).

En la bibliografía especializada sobre las relativas españolas y los FOMs destaca un estudio de Leonetti y Escandell (2017). Estos lingüistas sugieren que la aserción es clave para distinguir los dos tipos de relativas, y arguyen que un sujeto posverbal no puede interpretarse como foco informativo $^{3}$ en las relativas especificativas debido precisamente a que esta categoría discursiva 
es un FOM y, por tanto, queda excluida por el carácter presuposicional de estas relativas. Los ejemplos de (10) muestran el contraste entre oraciones matrices y relativas especificativas en cuanto a la posible interpretación como foco informativo del sujeto posverbal (Leonetti y Escandell 2017: 436):
a. Alquiló el apartamento Ernesto.
b. El apartamento [que alquiló Ernesto]

Ambas oraciones son gramaticales pero solo en la oración matriz (10a) se da la interpretación de foco del sujeto posverbal.

Los datos que discuto en este trabajo tendrán en cuenta otro subtipo de foco, como es la ANeg4. Ya ilustré en (7-9) la naturaleza de FOM de este categoría informativa. La pregunta de investigación a la que responderé será por tanto: ¿Es la ANeg realmente un FOM tanto en inglés como en español? ¿Se reflejará alguna diferencia entre ambas lenguas en relativas tanto explicativas como especificativas? Como argüía antes, estas preguntas vienen motivadas por la existencia de una diferencia clara entre el español y el inglés con respecto a la ANeg en otros contextos sintácticos, tal como he ilustrado en ejemplos previos.

La hipótesis inicial será que en inglés las especificativas no pueden albergar fenómenos como la ANeg, mientras que las explicativas sí; por el contrario, en español la ANeg estará permitida en los dos tipos de relativas. La raíz del problema reside en la distinción entre aserción y presuposición, cuestión que ya he mostrado como crucial para entender la compatibilidad de la ANeg y otros tipos de subordinadas. Sin embargo, un análisis semántico solo podrá dar cuenta de los datos del inglés. Para comprobar el nivel de aceptabilidad, he llevado a cabo un experimento en el que participaron 49 hablantes nativos de inglés y 61 de español.

Una explicación puramente semántica no puede dar cuenta de esta diferencia paramétrica. En mi análisis propongo una explicación sintáctica que gira en torno a dos ingredientes principales: 1) la herencia de rasgos discursivos por parte de T(iempo) desde el C(omplementante), que caracteriza a lenguas como el español frente al inglés (Miyagawa 2017, Jiménez-Fernández 2018, Ojea 2019), y 2) la presencia de un operador factivo (dotado del rasgo interpretable [Presuposición]) por encima de Sintagma de Tiempo (ST), el cual se mueve al Sintagma de Fuerza en las relativas especificativas, y la ausencia de dicho operador en relativas explicativas. La explicación de la diferencia entre los datos del inglés y del español la derivaré de la distinta posición que ocupa el constituyente negativo movido.

El trabajo se estructura de la siguiente manera. En la sección 2 presentaré la diferencia entre relativas especificativas y explicativas en términos de presuposición y aserción. En la sección 3 describiré el experimento y los resultados del mismo para comprobar si se valida mi hipótesis inicial. El apartado 4 incluye la propuesta formal de la derivación de la ANeg en ambos tipos de relativas en inglés y español. Por último, en la sección 5 concluiré el trabajo aportando problemas de investigación que deberán resolverse en futuros trabajos. 


\section{TIPOS DE RELATIVAS Y FENÓMENOS DE ORACIÓN MATRIZ}

Es un hecho ampliamente reconocido en las gramáticas descriptivas el distinguir entre oraciones relativas especificativas y explicativas (Quirk et al. 1985, Huddleston y Pullum 2002, Brucart 1999, entre otros). Radford (2019), a propósito del inglés, ilustra ambas clases de relativas en los ejemplos de (11):

(11) a. The allegations [which/that/ø Trump made during his campaign] turned out to be fake.

b. These allegations, [which/*that $/{ }^{*} \varnothing$ Trump made during his campaign], turned out to be fake.

(Radford 2019: 7, ejs. 1a-b)

Desde un punto de vista semántico, la relativa de (11a) es especificativa, y, como tal, restringe la clase de entidades denotada por el antecedente a aquellas que tienen la propiedad descrita en la relativa (Radford 2019: 7). Es decir, la relativa especificativa aporta información para identificar al antecedente en un contexto dado. Por el contrario, en (11b) la relativa es explicativa, y, por tanto, ofrece información adicional sobre el antecedente, la cual no es necesaria para su identificación.

En el plano discursivo, como he destacado antes, los dos tipos de relativa difieren entre sí con respecto a su conexión con el Fondo Común (FC). La información que dan las especificativas es ya parte del FC y, en consecuencia, este tipo expresa presuposición. Recordemos que la presuposición indica información ya compartida en el contexto. En clara oposición, las explicativas no pertenecen al FC y, por tanto, expresan aserción, siendo el contenido de las mismas nuevo en el contexto (Leonetti y Escandell 2017, Antomo 2012, Gärtner 2000). Si esta diferencia en términos informativos es correcta, los dos tipos de relativas constituyen dos contextos sintácticos perfectos para comprobar la posible legitimación de FOM. En concreto, si las relativas explicativas son asertivas y los FOM requieren aserción, podemos predecir que las explicativas, pero no las especificativas, serán compatibles con $\mathrm{FOM}^{5}$. A continuación ofrezco ejemplos que conducen a validar esta predicción:

(12) *The car that only rarely did I drive is in excellent condition.

'El coche que solo alguna vez conduje está en condiciones excelentes'.

(Hooper y Thompson 1973: 489, ej. 199)

(13) *These are the students to whom, this book, I will recommend.

'Estos son los estudiantes a los que este libro se lo recomendaré'.

(Haegeman 2012: 27, ej. 31a) 
(14) *The students that only at weekends did I see are living in the country now.

'Los estudiantes a los que solo en los fines de semana veía están viviendo en el campo'.

(Emonds 1976: 29, ej. 21)

(15) Hal, who under no circumstances would I trust, asked for a key to the vault.

'Hal, en quien bajo ningún concepto confiaría, pidió una llave de la caja fuerte'.

(Hooper y Thompson 1973: 472, ej. 41)

(16) This car, which only rarely did I drive, is in excellent condition.

'Este coche, que solo en ocasiones conduje, está en excelentes condiciones'.

(Hooper y Thompson 1973: 489, ej. 198)

(17) It's a demonstration of modern football, where time, you just don't get.

'Es una manifestación de fútbol moderno, donde tiempo simplemente no tienes'.

(Radford 2019: 20, ej. 33c)

(18) This is purely an admin charge by FedEx, which at no point did I agree to paying.

'Esto es meramente un cargo administrativo por parte de FedEx, que en ningún momento estuve de acuerdo en pagar'.

(Radford 2019: 20, ej. 33d)

Tanto la ANeg como la Topicalización se describen como FOM en inglés (Emonds 2004); de ahí que deban ser excluidos de oraciones presupuestas como las relativas especificativas de (12-14). Obsérvese que el antecedente de las relativas en estos ejemplos es definido, lo cual indica que la información expresada por la construcción "Sintagma Determinante + Relativa" tiene naturaleza de presuposición.

En lo que concierne a las relativas explicativas, se han definido como oraciones que expresan aserción. La predicción que podemos hacer es que, si la ANeg y la topicalización son FOM, estas deberían permitirse en las relativas explicativas. Los ejemplos de (15-18) avalan la validez de esta predicción6.

En cuanto al español, las relativas especificativas son totalmente compatibles tanto con la Topicalización o Dislocación a la Izquierda con Doblado de Clítico ${ }^{7}$ como con la ANeg, tal como ilustran los ejemplos (19-20), siempre que el contexto pragmático lo permita. Asimismo, las relativas explicativas españolas también pueden albergar estos FOM, tal como se aprecia en (2122).

(19) El coche que solo alguna vez he conducido está en excelentes condiciones, pero al de allá hay que hacerle un montón de reparaciones. 
(20) Los estudiantes que nunca antes se hayan leído este libro pueden sacarlo de la biblioteca.

(21) Este coche, que solo alguna vez he conducido yo, está en condiciones excelentes.

(22) Es toda una prueba de arte moderno, donde el tiempo no lo cuentan como horas trabajada.

Los ejemplos de (19-20) contienen relativas especificativas cuyo estatuto de presuposición es plenamente compatible con la ANeg, cuestión que no esperaríamos en un análisis puramente semántico. Esta afinidad resulta cuando menos intrigante, pues el inglés la rechaza en un contexto sintáctico idéntico. Volveré a este problema más adelante para ofrecer una solución a esta variación.

Por último, nótese que las explicativas en español se comportan exactamente igual que las del inglés, permitiendo tanto la ANeg como la Topicalización, como se observa en (21-22).

Como comentaba en la "Introducción", los datos del inglés son bastante paradójicos, pues unos autores consideran gramatical lo que otros ven como agramatical. Por otro lado, en español no tenemos ningún estudio sobre la compatibilidad de la ANeg en relativas. Por esta razón, es interesante realizar un experimento en el que los informantes confirmen o no la aceptabilidad de estas construcciones. La siguiente sección presenta este trabajo experimental y la discusión de los resultados.

\section{EL EXPERIMENTO Y LOS RESULTADOS}

En los estudios recientes de sintaxis teórica tiene un papel fundamental el uso de trabajos experimentales con el fin de que los datos que se barajen sean reales, y no los que ofrece el lingüista con su propio juicio de gramaticalidad (Ortega-Santos 2020, Sprouse y Almeida 2012, Sprouse et al. 2013). Muchos de estos estudios revelan la poca fiabilidad de los datos que se discuten en trabajos de corte teórico. Para paliar esta situación, es deseable que cualquier análisis teórico se base en datos experimentales que hayan sido comprobados estadísticamente. Por esta razón, antes de ofrecer mi propuesta formal sobre la compatibilidad de la ANeg en las relativas y comparar las dos lenguas objeto de estudio aquí, presentaré el diseño de la tarea experimental llevada a cabo con nativos de ambas lenguas y los resultados obtenidos.

\subsection{Metodología: tipo de informantes y diseño de la encuesta}

En ambas lenguas el grupo de informantes ha sido homogéneo. Tenían título universitario o eran estudiantes de universidad. En cuanto al origen de los participantes, para la encuesta en inglés, estos eran mayormente británicos (Inglaterra y Escocia), aunque también tomaron parte algunos estadounidenses (California) e incluso neozelandeses. En la encuesta española, los 
participantes eran mayormente españoles (Andalucía y Cataluña) y argentinos, aunque también participaron informantes de Venezuela, Ecuador y México.

La encuesta se diseñó mediante la herramienta Google Forms. Contenía 18 ítems, de los que 6 ilustraban relativas especificativas, otros 6 contaban con relativas explicativas, y los 6 restantes eran distractores o fillers. Los ejemplos habían sido convenientemente mezclados de forma aleatoria para evitar cualquier creación de un patrón de respuesta por parte de los informantes.

En las instrucciones se les pedía que dieran su juicio sobre las oraciones. Para las respuestas, se les proporcionaba una Escala Likert de 7 opciones, donde 1 significaba "totalmente inaceptable, nunca la usaría" y 7 indicaba "totalmente aceptable y natural". El número de respuestas obtenido fue de 49 para el cuestionario inglés y de 61 para el español.

Todos los ítems estaban precedidos por un contexto que facilitara la lectura de foco del elemento negativo antepuesto, que era bien un argumento, bien un adjunto dentro de la relativa. A continuación muestro dos ejemplos del español, uno con una relativa explicativa (23) y el otro con una especificativa (24):

[Contexto: Una pareja está planeando un viaje a un lugar exótico, donde podría haber conflictos locales. Él le dice a ella:]

(23) En India, donde a ningún turista han secuestrado esos terroristas, hay varios destinos que me atraen.

(24) En Asia podemos visitar aquellos países en los que a ningún turista hayan secuestrado esos terroristas.

\subsection{Resultados y discusión}

En esta sección voy a analizar los resultados de la encuesta lingüística, empezando por las explicativas en inglés y en español. Las relativas explicativas fueron testadas mediante oraciones como las de (25) en inglés y (26) en español:

[Contexto: Un profesor se queja del poco éxito que tiene su asignatura entre los estudiantes, después de que otro profesor le dijera que había oído que la sintaxis gozaba de gran popularidad especialmente entre los estudiantes Erasmus]

(25) Syntax, which only very rarely do foreign students take, is not very popular among local students either.

(26) Sintaxis 1, en la que rara vez se matriculan estudiantes Erasmus, no es tampoco muy popular entre los alumnos españoles. 
Los resultados obtenidos en el experimento son los que aparecen en los gráficos 1 y 2 :

49 respuestas

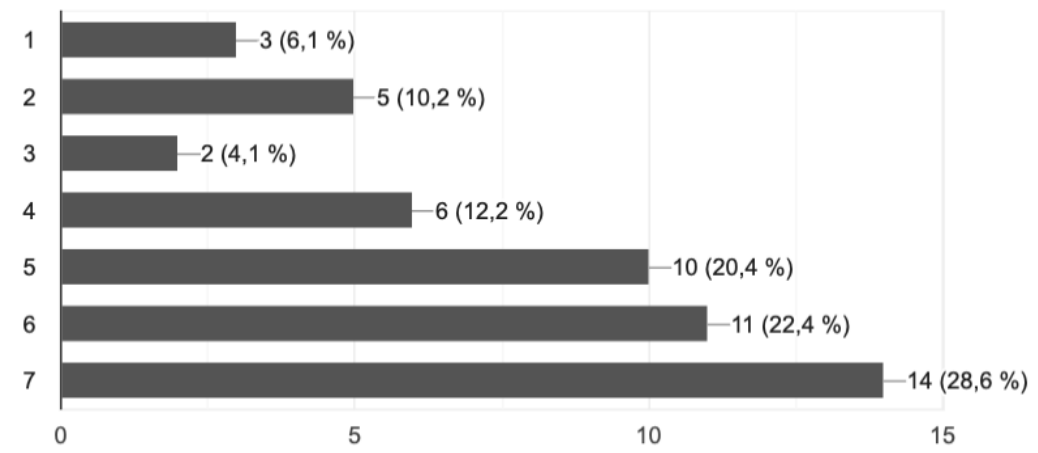

Gráfico 1. Resultados ANeg en explicativas en inglés

61 respuestas

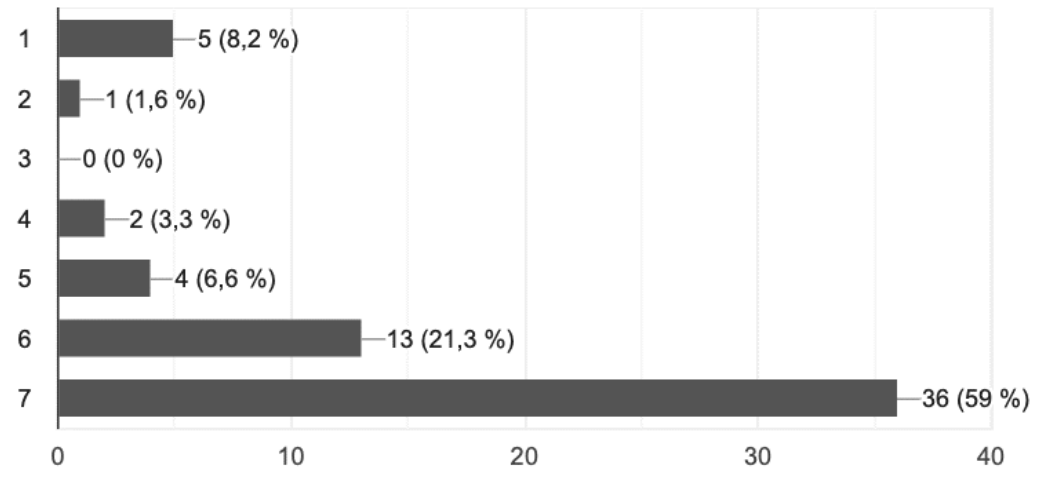

Gráfico 2. Resultados ANeg en explicativas en español

Para comparar los resultados de las encuestas, he fijado el umbral de la gramaticalidad en 5. Es decir, aquellas construcciones a las que los participantes otorgaron una valoración de 5 a 7 las he considerado gramaticales. En el gráfico 2, podemos observar que en español la ANeg es claramente compatible con las relativas explicativas. Los informantes mayormente eligieron las opciones 5-7, lo cual supone que aproximadamente un $76 \%$ juzgaron aceptable el uso de este fenómeno en las explicativas. Por tanto, ejemplos como el de (26), donde el adverbial negativo rara $v e z$ se antepone en una relativa explicativa, son plenamente gramaticales para los informantes.

Nótese que en este caso se antepone un adverbial. No obstante, otros ejemplos, como el de (24), incluyen el movimiento de un argumento (a ningún turista). El gráfico 2 refleja ambos supuestos, apoyando la idea de que la ANeg tanto de argumentos como de adjuntos es lícita en las relativas explicativas del español. 
Por otro lado, si observamos el gráfico 1, podemos apreciar que en inglés los encuestados seleccionaron también las opciones 5-7 en su mayoría, dando un total de $71 \%$ de hablantes que consideraron aceptable la construcción. Estos resultados del gráfico 1 recogen el movimiento de un adjunto, como en (25), y de un argumento, como en (23). De aquí inferimos que la primera parte de la hipótesis inicial queda validada, pues los datos arrojan la conclusión de que, tanto en inglés como en español, las relativas explicativas permiten la ANeg.

Veamos ahora qué ocurre con las relativas especificativas. Las oraciones testadas en esta tipología incluían relativas especificativas como las ejemplificadas en (27) para el inglés y en (28) para el español, con un argumento prepuesto, y del tipo de las de (29) para el inglés y (30) para el español, con un adverbial antepuesto:

[Contexto: Dos estudiantes de diferente curso están hablando sobre distintas asignaturas del Grado, y el de curso superior le dice al otro estudiante de curso inferior:]

(27) This is the course in which no student has the teacher ever given a grade higher than B to.

(28) Esta es la asignatura en la que a ningún estudiante le da el profesor más de un 8.

[Contexto: Un vendedor de coches de segunda mano está intentando vender uno a unos clientes]

(29) The car which only with great reluctance did he agree to sell is the Maserati.

(30) El coche que solo alguna vez ha conducido su dueño está en excelentes condiciones, pero al de allá hay que hacerle un montón de reparaciones.

Los resultados de la ANeg en relativas especificativas se muestran en los gráficos 3 y 4 :

49 respuestas

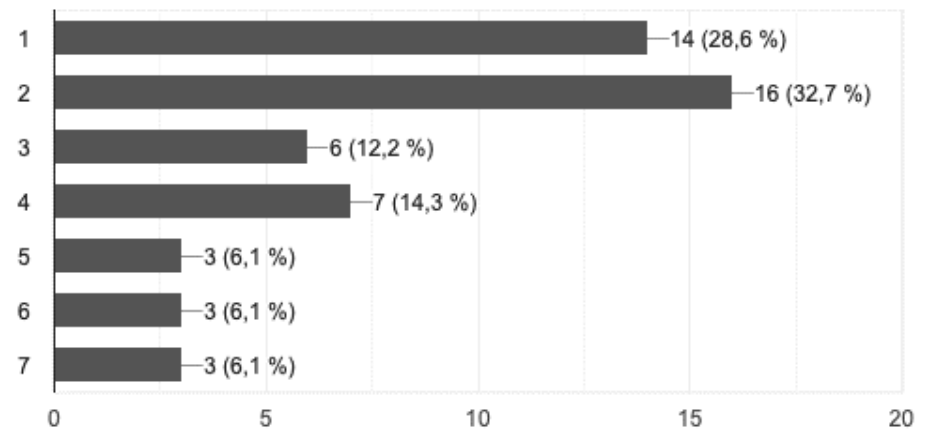

Gráfico 3. Resultados ANeg en especificativas en inglés 


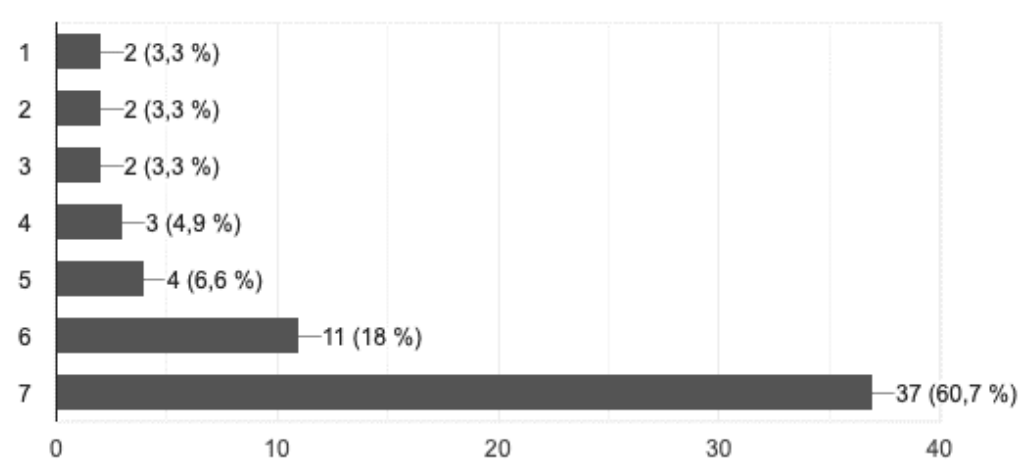

Gráfico 4. Resultados ANeg en especificativas en español

A juzgar por los gráficos 3 y 4, es en las especificativas donde surge una clara distinción entre el inglés y el español. El inglés arroja el dato de solo un $18 \%$ de gramaticalidad, frentre a un $43 \%$ de agramaticalidad. Este resultado aúna de nuevo tanto la anteposición de argumentos como la de adjuntos. Claramente, la ANeg no es viable en especificativas en inglés. Ahora bien, en español observamos algo muy interesante. La suma de las opciones 5-7 nos da un total de $85 \%$ de gramaticalidad, teniendo en cuenta la anteposición de argumentos y adjuntos. Este dato valida completamente la hipótesis inicial, según la cual se esperaba que las especificativas admitieran la ANeg en español.

El principal resultado empírico que se ha obtenido muestra que el inglés distingue en términos de aceptabilidad entre los dos tipos de relativas cuando se da la ANeg, mientras que en español se legitima este fenómeno en ambas clases de relativas. Como ya he señalado, si nos basamos en la distinción entre aserción y presuposición en el plano de la semántica, los datos del español no se podrían explicar. Por esta razón, en la siguiente sección argüiré que un análisis sintáctico, que tenga en cuenta la distinción semántica entre aserción y presuposición, tendrá mucho más poder explicativo que un análisis puramente semántico.

\section{HACIA UN ANÁLISIS SINTÁCTICO-DISCURSIVO DE LA ANEG EN RELATIVAS}

Como ya he avanzado, necesitamos un análisis formal que explique el paralelismo entre el inglés y el español en cuanto a las relativas explicativas, a la vez que dé cuenta de la variación identificada en las relativas especificativas. Dado que mi propuesta asume mecanismos sintácticos como la herencia de rasgos, la existencia de un operador eventivo o factivo en las oraciones presupuestas, la configuración cartográfica de la periferia izquierda y los efectos de intervención, voy a describir cada uno de estos conceptos en primer lugar. De esta manera, la propuesta formal quedará más clara. 


\subsection{Conceptos previos}

\subsubsection{La configuración cartográfica de la oración}

Desde Rizzi (1997), los fenómenos discursivos como la anteposición de tópico y foco han ocasionado una robusta línea de investigación de la periferia izquierda de la oración, denominada cartografía. En esta aproximación lingüística se parte de la idea de que el Complementante (C) se puede dividir en núcleos más específicos dotados de un rasgo discursivo que atraerá a un determinado constituyente sintáctico para su validación.

A continuación represento la cartografía de la oración, reduciéndola a aquellas proyecciones que usaré en mi propuesta $(\mathrm{Top}=\text { Tópico; Foc }=\text { Foco; } \mathrm{T}=\text { Tiempo; } \mathrm{V}=\mathrm{Verbo})^{8}$ :

$$
\text { [sfuerza Fuerza [sTop Top [sfoc Foc [sт T [sv V...]]]]] }
$$

Esta configuración da cuenta de la derivación sintáctica de oraciones como la subordinada de (32), representada en (33):

(32) Dijo que ese libro a nadie se lo regalaría.

(33) [sFuerza que [sTop ese libro Top [sFoc a nadie Foc [sT se lo regalaría [sv regalaría

ese libro a nadie]]]]

El núcleo Fuerza está ocupado por el Complementante que, tipificando la oración como declarativa. El objeto ese libro se mueve al STop, pues es el tópico de la oración, dejando en su lugar original una copia nula (Chomsky 2008), aunque recuperada en español por el clítico resuntivo lo. De igual forma, el objeto a nadie se desplaza al SFoc, pues es el foco, y también deja una copia nula en su lugar original. Por último, el verbo con los clíticos se mueve al núcleo T (Suñer 1992). En mi propuesta haré uso de las proyecciones SFuerza, SFoc y ST y reformularé el sitio que ocupan las categorías discursivas en español e inglés.

\subsubsection{Herencia de rasgos}

Chomsky (2008) propone que las categorías funcionales como C entran en la derivación sintáctica dotadas de rasgos gramaticales no interpretables que se han de borrar antes de enviar esta derivación a las interfaces (componente semántico y componente fonológico). Para borrar un rasgo, este debe estar validado con otro mediante el mecanismo conocido como Concordancia. Así, por ejemplo, $\mathrm{C}$ tiene rasgos de número y persona que en inglés y español son heredados por $\mathrm{T}$, 
dando cuenta de por qué el sujeto en inglés debe siempre ocupar la posición de especificador del ST.

Sin embargo, $\mathrm{C}$ también puede contener rasgos discursivos que, dependiendo de cada lengua, serán heredados por T o los retendrá C (Miyagawa 2010). Para el inglés, se ha propuesto que estos rasgos discursivos (como [tópico] y [foco]) no bajan a T, sino que se quedan en C. De ahí que, si hay un movimiento discursivo, este tendrá como destino un especificador en el área de C. La oración en (2), repetida aquí como (34), tendría la estructura de (35):

(34) No other colleague would he turn to. (Radford 2009: 329)

'A ningún compañero recurriría yo'.

(35) [sFuerza $\varnothing$ [sFoc no other colleague [would] [sт he would turn to no other colleague]]]

Sin embargo, en otras lenguas como el español o el japonés, se ha propuesto que al menos ciertos rasgos discursivos bajan de $\mathrm{C}$ a $\mathrm{T}$ y, consecuentemente, fenómenos como la ANeg conllevan el movimiento del elemento focalizado al especificado de T, como ocurre en (37), representación de la oración en (1), repetida a quí como (36):

(36) De ninguno de esos problemas trató la reunión. (Bosque 1980: 25)

(37) [scomp Ø [sт de ninguno de esos problemas trató [sv la reunión trató de ningune de esos problemas]]]

Para validar el rasgo [foc] que contiene T por herencia desde C, el constituyente negativo se mueve al especificador de T dejando una copia en su lugar original (Jiménez-Fernández 2018).

\subsubsection{Efectos de intervención}

En la tradición de la gramática generativa los efectos de intervención han sido un concepto recurrente para explicar ciertas malas formaciones sintácticas. Las definiciones de estos efectos son muchas y variadas (Rizzi 1990, 2004, 2006; Haegeman 2012; Villata et al. 2016). En general, se concibe que un constituyente no se puede mover a una posición si entre ambos lugares existe un elemento que también podría desplazarse a la misma posición.

Una de las versiones de efectos de intervención consiste en la distinción que establece Richards (1999) entre crossing chains (cadenas cruzantes) y nesting chains (cadenas anidantes). Las cadenas cruzantes solo se permiten en movimientos multiples a Especificadores Múltiples de un único núcleo, mientras que las cadenas anidantes pueden darse entre especificadores de distintos núcleos. A continuación, represento un caso de cadena cruzante lícito en especificadores múltiples 
de un mismo núcleo, un caso anidante lícito en especificadores de distintos núcleos y otro caso de cadena cruzante ilícito en especificadores múltiples:

(38) Cruzante lícito

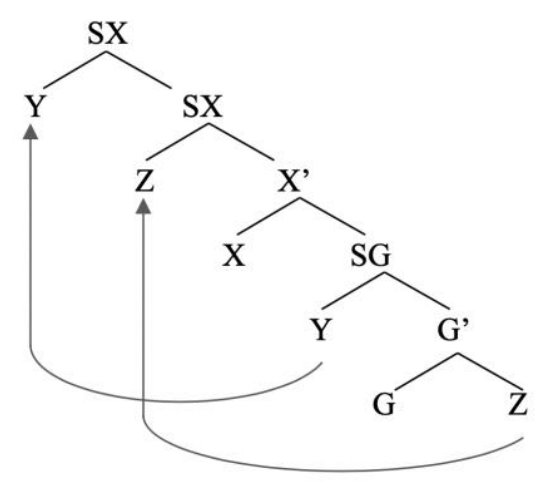

(40) Cruzante ilícito

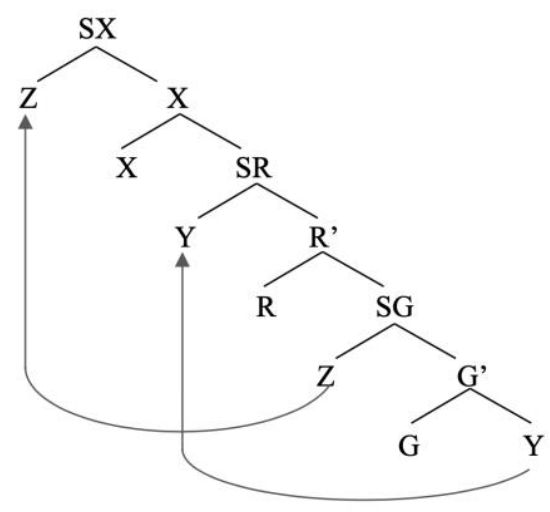

(39) Anidante lícito

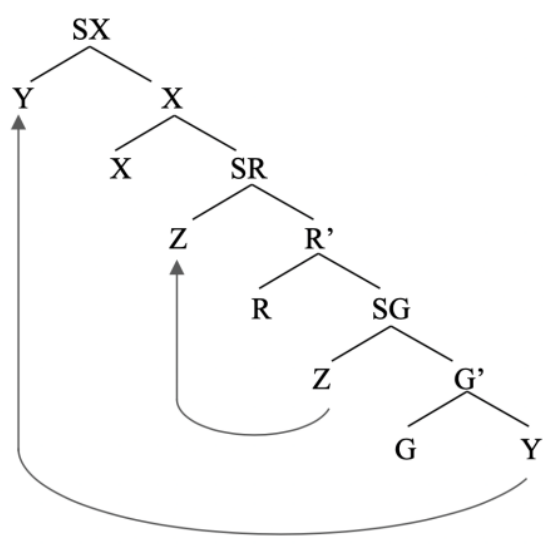

Como expondré en mi propuesta, el hecho de que los distintos movimientos que se producen en las relativas den lugar a cadenas de un tipo u otro será crucial para explicar la variación paramétrica detectada en español e inglés.

\subsubsection{Sintactización de la dicotomía aserción/presuposición}

El último concepto que discutiré de cara a presentar mi propuesta sobre las relativas consiste en el reflejo sintáctico de la distinción entre presuposición y aserción. En la bibliografía generativista actual hay un debate muy fructífero sobre si ciertas cuestiones que pertenecen al discurso se proyectan también en la sintaxis. Así, por ejemplo, cuestiones como la orientación hacia el oyente o hablante de los marcadores discursivos han motivado la proyección de ciertos sintagmas con un contenido claramente discursivo (Speas 2000, Speas y Tenny 2003, Haegeman y Hill 2013, Portner et al. [en prensa], entre otros). 
En esta misma línea, una propiedad de estructura informativa como la expresión de la aserción y la presuposición se ha reflejado en la derivación sintáctica mediante el postulado de proyecciones o rasgos relacionados con la aserción. Por ejemplo, Alboiu y Hill (2019), siguiendo a Meinunger (2004), proponen un Operador Asertivo en el especificador de SComp o SFuerza para tipificar la oración como asertiva.

Basándome en propuestas anteriores de Leonetti y Escandell (2017), Hooper y Thompson (1973), Jiménez-Fernández (2018), Haegeman (2012), Gärtner (2000) y Antomo (2012), entre otros, para los que la diferencia entre los dos tipos de relativas radica en que las especificativas expresan presuposición, mientras que las explicativas indican aserción, propongo la proyección de un núcleo Fuerza no asertivo en las relativas especificativas, que establece una relación de Concordancia con un operador factivo o eventivo que se genera en el especificador de un Sintagma Funcional (SF) (Miyagawa 2017, Jiménez-Fernández y Miyagawa 2014). De esta manera, la oración queda tipificada como presupuesta. Por el contrario, en las relativas explicativas no habrá operador factivo o no asertivo, tipificándose la oración como aserción por defecto.

Por último, siguiendo a Radford (2019), asumiré que los operadores relativos se mueven al especificador de un núcleo Relativo (Rel), que se proyecta por encima de Fuerza.

\subsection{Análisis de ANeg en relativas y comparación del inglés y el español}

Una vez que hemos introducido todos los ingredientes que van a interactuar en el análisis de las relativas, paso a detallar la derivación que propongo para los dos tipos de relativas en inglés y en español, partiendo de los resultados empíricos del experimento. Recordemos que la ANeg es plenamente gramatical en las relativas explicativas en inglés, frente a su agramaticalidad en las especificativas, mientra que el español permite la ANeg en ambos tipos de relativas.

Seguidamente expongo el análisis de las especificativas en inglés:

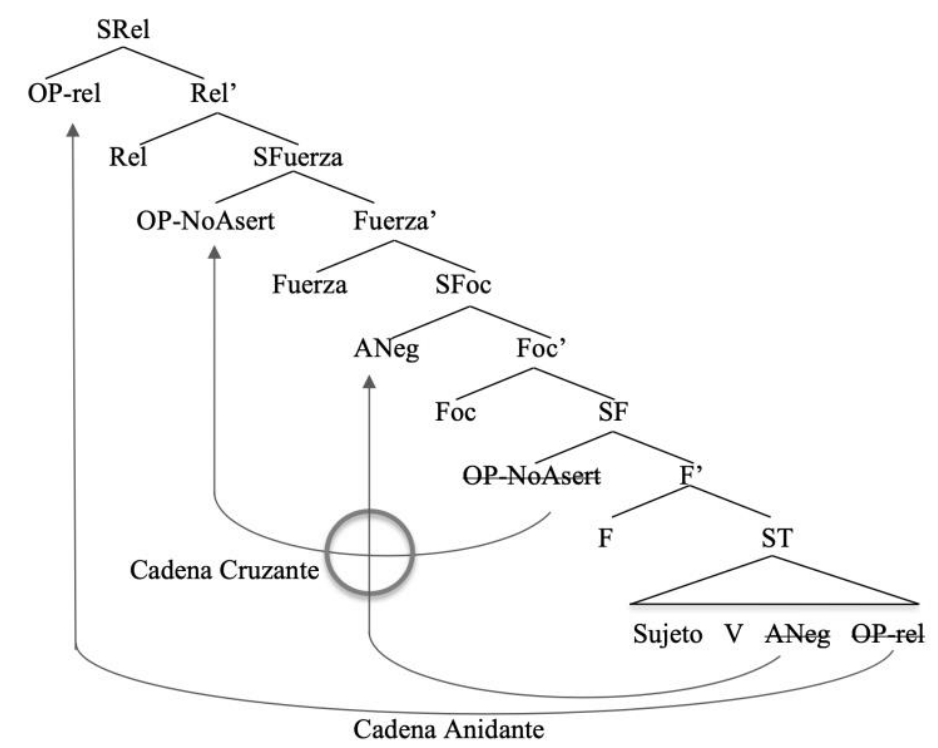


El elemento negativo (ANeg) entra en la derivación con un rasgo [foc] interpretable. Como los rasgos discursivos del inglés permanecen en $C$ (en este caso en Foc), ANeg se desplaza al especificador de SFoc. Por otro lado, el Operador no asertivo que se genera en el SF se ha de mover al especificador del SFuerza para tipificar la oración como presupuesta. Estas dos cadenas producen un efecto de intervención (señalado mediante el círculo), pues se cruzan entre sí al dirigirse a especificadores de núcleos diferentes (Richards 1999). Este cruce explica por qué la ANeg es imposible en una relativa especificativa en inglés.

El efecto de intervención que se observa en la derivación de las especificativas en (41) explica el porcentaje de agramaticalidad que arroja el experimento expuesto en la sección 3.2 (gráfico 3). Esta explicación teórica predice la degradación de ejemplos como (27) y (29) del inglés, tal como hemos visto.

Nótese que la cadena del Operador Relativo no ofrece problemas, pues es anidante y no cruza ninguna otra cadena. Esto da cuenta de que si evitamos la ANeg en una relativa especificativa el resultado sí es gramatical, tal como se aprecia en (42), correspondiente al ejemplo agramtical de (29) pero dejando el constituyente negativo en su posición original.

(42) The car which he agreed to sell only with great reluctance is the Maserati.

El coche que él estuvo de acuerdo en vender solo con cierto recelo es el Maserati'.

De nuevo este tipo de ejemplos donde no hay movimiento del elemento negativo apoya el hecho de que la explicación de la agramaticalidad en los ejemplos de especificativas inglesas donde sí ha habido ANeg no pueda ser semántica, sino más bien sintáctica.

Paso a continuación a presentar la derivación de las relativas especificativas españolas, que como hemos confirmado son compatibles con la ANeg:

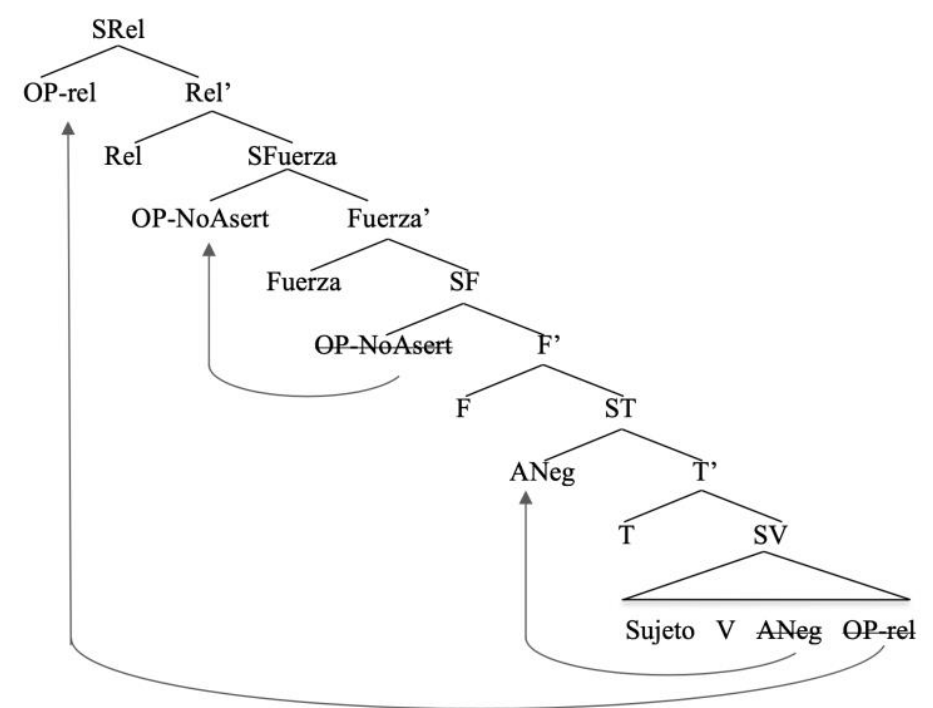

En los resultados del experimento quedaba exponencialmente claro que las especificativas españolas sí permitían la ANeg. Para explicar esta variación con respecto al inglés, en mi propuesta 
el rasgo discursivo [foc] lo hereda T en español, provocando un cambio radical en la derivación de la ANeg, pues el elemento negativo se mueve al especificado de ST. Este desplazamiento no acarrea ninguna clase de cadena cruzante con respecto al Operador no asertivo, tal como se observa en (43). La consecuencia inmediata es que la construcción es gramatical en contextos de presuposición como las relativas especificativas. Es decir, la razón por la que la ANeg es lícita o ilícita radica en la sintaxis y no en la semántica.

Esta propuesta explica por qué los ejemplos de especificativas españolas (28) y (30) en el experimento alcanzan unas cifras tan altas en lo que concierne a su gramaticalidad. Centrémonos en (28), repetida aquí como (44):

(44) Esta es la asignatura en la que a ningún estudiante le da el profesor más de un 8.

En esta relativa especificativa el complemento indirecto a ningún estudiante se ha movido al especifcador de ST sin llegar a cruzar la cadena del operador no asertivo. Por otro lado, el operador relativo también se mueve, pero a una posición más alta (especificador de Rel). El resultado es legítimo porque las cadenas obtenidas son anidantes, tal como observamos en (43), lo cual da cuenta del porcentaje del 85 \% que arroja el gráfico 3.

Pasamos seguidamente a las relativas explicativas, las cuales aceptan la ANeg tanto en español como en inglés. Recordemos que este es un contexto de aserción y, por tanto, en la derivación sintáctica no habrá ningún operador factivo. Esto facilitará todo el proceso. La única diferencia entre las dos lenguas consiste en la posición que ocupará la ANeg según si la lengua requiera la herencia del rasgo [foc] por parte de T o no. Muestro las derivaciones en inglés y en español en (45) y (46), respectivamente:

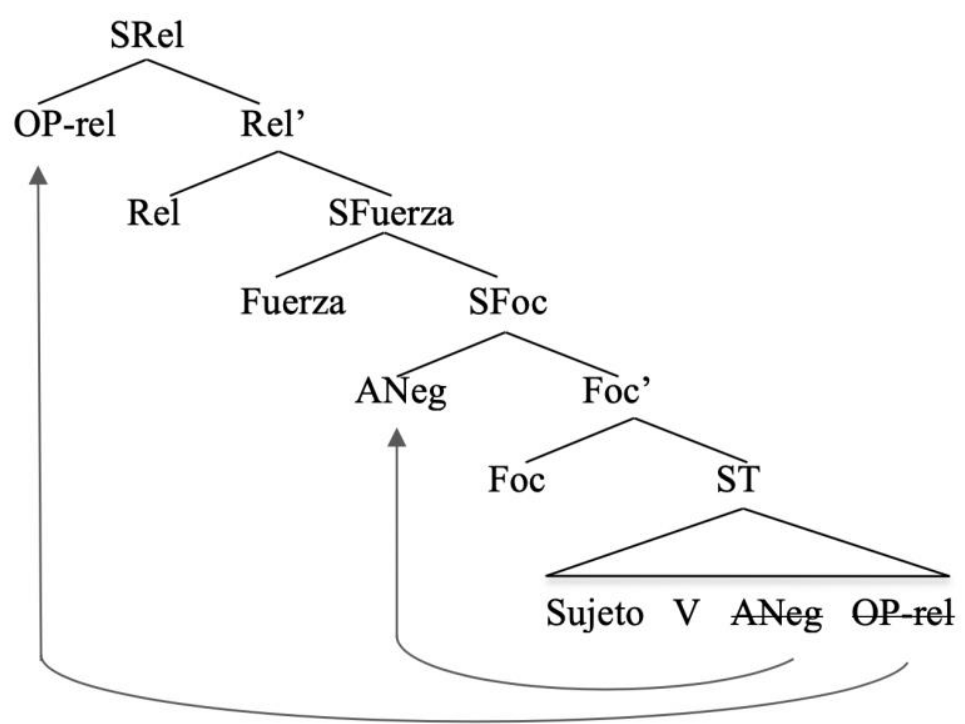


(46)

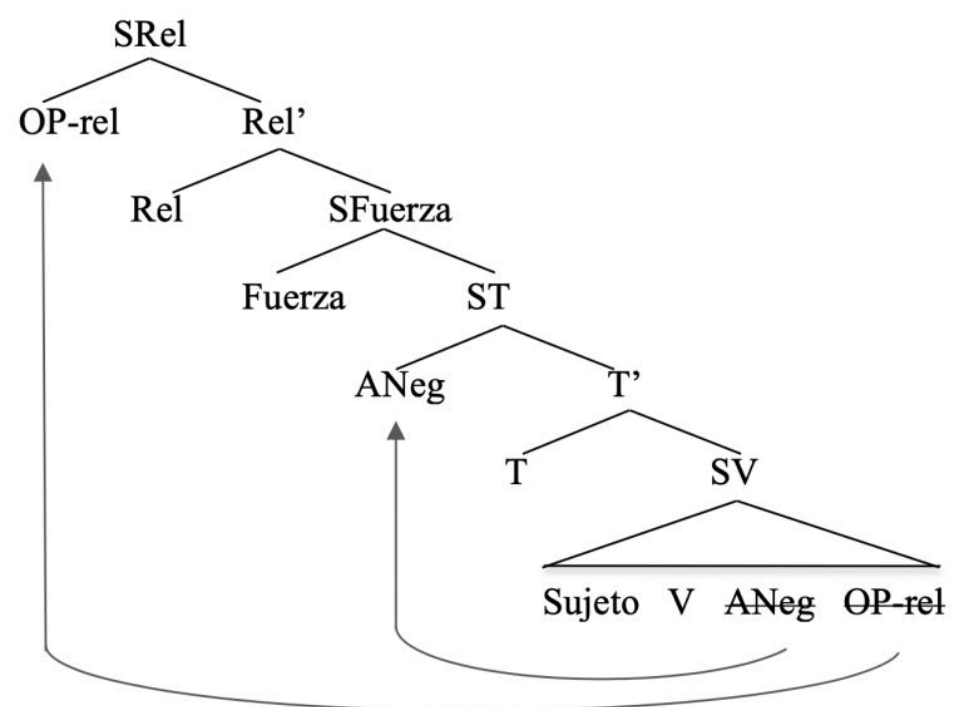

Tanto en la derivación inglesa como en la española observamos que las cadenas que los movimientos comportan son anidantes, lo cual no acarrea ningún tipo de intervención sintáctica. Como única distinción, podemos ver que la ANeg ocupa el especificador de SFoc en inglés y el de ST en español, precisamente debido a la herencia del rasgo [foc] desde el área del C hasta $\mathrm{T}$ en español, tal como he propuesto anteriormente. Estas dos derivaciones están bien formadas y, por tanto, las construcciones son plenamente gramaticales, como vemos en (25) y (26), repetidas como (47) y (48):

(47) Syntax, which only very rarely do foreign students take, is not very popular among local students either.

(48) Sintaxis 1, en la que rara vez se matriculan estudiantes Erasmus, no es tampoco muy popular entre los alumnos españoles.

En ambos casos los datos arrojan cifras realmente altas en los gráficos 1 y 2 . El $71 \%$ de los encuestados para el inglés y el $76 \%$ de los informantes para el español consideraron que la ANeg en oraciones relativas explicativas es totalmente lícita, lo cual es esperable si, tal como he propuesto, las cadenas resultantes en ambas lenguas son anidantes.

Para concluir este apartado, he propuesto que las relativas explicativas no proyectan ningún tipo de operador no asertivo, mientras que las especificativas sí, de manera que podamos formalizar la diferencia entre aserción y presuposición que también se da en las relativas. Por otro lado, he sugerido que el rasgo discursivo [foc] se queda en el SComp en inglés, mientras que en español baja a ST. Esto hace que, cuando queramos generar una relativa especificativa con ANeg, tengamos que fijarnos en si la lengua va a determinar que las cadenas creadas por los distintos movimientos se crucen unas con otras. En inglés la cadena de la ANeg cruza la del operador factivo, 
haciendo que la construcción sea agramatical. Por el contrario, en español, estas cadenas son anidantes y, por tanto, las oraciones resultantes son perfectamente gramaticales.

\subsection{Un argumento a favor del análisis: ligamiento cuantificador}

En esta última parte, voy a presentar evidencia empírica que apoye el análisis detallado en la sección precedente, basándome en el ligamiento de cuantificadores (quantifier binding), según el cual una anáfora puede estar ligada en su dominio local a un antecedente que contenga un cuantificador (ningún, algún, etc.). Para que se cumpla esta condición, este antecedente cuantificador debe ocupar una posición argumental como la de especificador de ST (frente a una posición no argumental como la del especificador de SComp o de una de sus proyecciones) -consúltese al respecto Lasnik (2003), López (2009) y Miyagawa (2010)—.

Veamos esta relación de ligamiento cuantificador en una oración matriz como la de (49), adaptada de Jiménez-Fernández (2018: 33):

(49) [A ninguno de los hermanos $]_{i}$ le ha dicho sui madre que llegara más temprano.

En esta oración el constituyente negativo a ninguno de los hermanos se ha antepuesto y ocupa el especificador de ST (según he propuesto). Desde esta posición el objeto directo liga al cuantificador posesivo su del sujeto su madre, de manera que sirve como antecedente del posesivo. En una relativa como la de (50) encontramos exactamente el mismo tipo de relación de ligamiento:

(50) Esa es la noticia que [a ninguno de los hermanos]i le comunicaría su madre sin echarse a llorar.

La interpretación en la que el elemento a ninguno de los hermanos puede ser el antecedente de la anáfora posesiva solo es posible si este último ocupa una posición interna al ST. Por tanto, estos datos apoyan la propuesta de mover el constituyente negativo al especificador de ST en español. Veamos qué ocurre en inglés. Por los resultados en (51) y (52), no se puede establecer la relación de ligamiento ni en oraciones matrices ni en relativas:

(51) *[To none of the siblings]i did theiri mother say that they should arrive earlier.

(52) *These are the news which [to none of the siblings]i did their $i_{i}$ mother give.

En ambas construcciones el elemento negativo ocupa una posición no argumental desde la que no puede ligar una anáfora. En coneecuencia, (51-52) apoyan el movimiento del constituyente negativo al área del SComp. Por último, la pasiva inglesa conlleva el movimiento del sujeto al especificador de ST, posición argumental. La predicción es que, si en vez de ANeg usamos una 
pasiva en inglés, el resultado es gramatical, lo cual queda validado a tenor de ejemplos como el de (53):

(53) [None of the siblings] i were told by theiri mother to arrive earlier.

Estos datos apoyan la idea de que algunos rasgos discursivos del español los hereda T, el cual atrae al constituyente negativo mediante la ANeg. Sin embargo, estos rasgos se quedan en el área del SComp en inglés, forzando al elemento negativo a moverse a una posición no argumental desde la que no puede ligar a una anáfora.

\section{CONCLUSIONES Y FUTURAS LÍNEAS DE INVESTIGACIÓN}

En este trabajo he mostrado que las oraciones relativas exhiben un comportamiento diferente interlingüísticamente con respecto a Fenómenos de Oración Matriz como la ANeg. Las relativas explicativas en inglés y español permiten la ANeg ya que son muy similares a las oraciones matrices en el sentido de que expresan aserción. Por el contrario, las relativas especificativas expresan presuposición, lo cual en un análisis semántico se traduce como incompatibilidad de los FOM con este tipo de estructuras. Sin embargo, hemos observado y comprobado experimentalmente que las restrictivas inglesas no permiten la ANeg, mientras que las restrictivas españolas sí, apuntando hacia un análisis sintáctico que dé cuenta de las diferencias paramétricas.

Para explicar la variación entre el inglés y el español, he propuesto un análisis teórico basado en la proyección de un OP no asertivo cuya cadena cruza la cadena de la ANeg cuando el elemento negativo se mueve al SFoc en inglés. No obstante, no hay ningún cruce o intersección en español, ya que la ANeg conlleva movimiento al ST, el cual está más bajo que el OP no asertivo. Este análisis sintáctico es superior al puramente semántico por el alcance de su poder explicativo, ya que da cuenta de los entresijos gramaticales que encierran los dos tipos de relativas que he estudiado aquí.

Varias preguntas surgen a tenor del análisis que he propuesto. La distinción entre aserción y presuposición atañe también a las subordinadas. Por un lado, sabemos que una subordinada seleccionada por un verbo o un adjetivo puede ser asertiva o presupuesta (Jiménez-Fernández y Miyagawa, 2014). La pregunta que esto conlleva es si también el español y el inglés muestran diferencias con respecto a la compatibilidad de la ANeg. También nos podemos preguntar qué ocurre en las subordinadas adverbiales. Haegeman (2012) propone la clasificación de las adverbiales en dos tipos: las centrales y las periféricas. Estas últimas parecen asemejarse a las oraciones matrices, pero ¿pueden FOM como la ANeg emplearse en las adverbiales periféricas? ¿Habría de nuevo variación paramétrica entre lenguas como el español y el inglés? El tema abre varias líneas de investigación que, si se combina la lingüística experimental y la teórica, pueden dar frutos que ayuden a entender el funcionamiento de la lengua. 


\section{Bibliografía}

AlbolU, G.; Hill, V. (2019): "Narrative infinitives, narrative gerunds, and the features of the C-T system", Journal of Historical Syntax 3, pp. 1-36.

Antomo, M. (2012): "Interpreting embedded verb second: Causal modifiers in German”, Proceedings of ConSOLE 17, pp. 27-51.

BATLlORI, M; HeRnANZ, M. L. (2014): "Weak focus and polarity: Asymmetries between Spanish and Catalan", en T. Biberauer y G. Walkden (eds.): Syntax over Time: Lexical, Morphological and InformationStructural Interactions. Oxford: Oxford University Press, pp. 280-298. https://doi.org/10.1093/acprof:oso/9780199687923.003.0017

BEnINCÀ, P. (2012): “Determiners and relative clauses”, Iberia: An International Journal of Theoretical Linguistics 4(1), pp. 92-109.

BENINCÀ, P.; CinQUE, G. (2014): “Kind-defining relative clauses in the diachrony of Italian”, en P. Benincà, A. Ledgeway y N. Vincent (eds.): Diachrony and Dialects. Oxford: OUP, pp. 257-78. https://doi.org/10.1093/acprof:oso/9780198701781.003.0013

BianChI, V.; FrasCARELli, M. (2010): “Is topic a root phenomenon?”, Iberia: An International Journal of Theoretical Linguistics 2, pp. 43-88.

BoSQUE, I. (1980): Sobre la Negación. Madrid: Cátedra.

BRUCART, J. M. (1999): “La estructura del Sintagma Nominal: Las oraciones de relativo”, en I. Bosque y V. Demonte (dirs.): Gramática descriptiva de la lengua española. Madrid: Espasa, vol. 1, pp. 395-522.

Сномsку, N. (2008): “On phases”, en R. Freidin, C. Otero y M. L. Zubizarreta (eds.): Foundational Issues in Linguistic Theory. Cambridge, MA: MIT Press, pp. 133-166.

Culicover, P. (1991): "Polarity, inversion, and focus in English", en Proceedings of the Eighth Eastern States Conference on Linguistics. Columbus: ESCOL Publications / Department of Linguistics, Ohio State University, pp. 46-68.

DE CAT, C. (2012): “Towards an interface definition of root phenomena” en L. Aelbrecht, L. Haegeman y R. Nye (eds.): Main Clause Phenomena. Amsterdam/Philadelphia: John Benjamins, pp. 135-158. https://doi.org/10.1075/la.190.06cat

EmondS, J. (1969): Root and Structure-Preserving Transformations. Tesis doctoral. Cambridge, MA: Massachusetts Institute of Technology.

EmONDS, J. (1976): A Transformational Approach to English Syntax. New York: Academic Press.

EmondS. J. (2004): “Unspecified categories as the key to root constructions”, en D. Adger, C. De Cat y G. Tsoulas (eds.): Peripheries: Syntactic Edges and their Effects. Dordrecht: Kluwer, pp. 75-121. https://doi.org/10.1007/1-4020-1910-6 4

FrasCARELLI, M.; JimÉNEZ-FERNÁNDEZ, Á. L. (2017): "Subextraction at the discourse-grammar interface: A featural approach to island effects”, en O. Fernández-Soriano, E. Castroviejo e I. Pérez-Jiménez (eds.): Boundaries, Phases, and Interfaces. Case Studies in Honor of Violeta Demonte. Amsterdam/Philadelphia: John Benjamins, pp. 224-254. https://doi.org/10.1075/la.239.11fra

FRASCARELLI, M.; JIMÉNEZ-FERNÁNDEZ, Á. L. (2019): “Understanding partiality in pro-drop languages: An information-structure approach”, Syntax 29(2/3), pp. 162-198. https://doi.org/10.1111/synt.12184 
Gallego, Á. (2007): Phase Theory and Parametric Variation. Tesis doctoral. Bellaterra, Barcelona: Universitat Autónoma de Barcelona.

GÄRTNER, H.-M. (2002): “On the force of V-2 declaratives”, Theoretical Linguistics 28, pp. 33-42. https://doi.org/10.1515/thli.2002.28.1.33

Haegeman, L. (2012): Adverbial Clauses, Main Clause Phenomena, and the Composition of the Left Periphery. Oxford: Oxford University Press. https://doi.org/10.1093/acprof:oso/9780199858774.001.0001

HaEgeman, L.; HILL, V. (2013): “The syntactization of discourse”, en R. Folli, C. Sevdali y R. Truswell (eds.): Syntax and its Limits. Oxford: Oxford University Press, pp. 370-390. https://doi.org/10.1093/acprof:oso/9780199683239.003.0018

HEYCOск, C. (2006): “Embedded root phenomena”, en M. Everaert y H. van Riemsdijk (eds.): The Blackwell Companion to Syntax. Oxford: Blackwell, vol. 2, pp. 174-209. https://doi.org/10.1002/9780470996591.ch23

HOOPER, J.; THOMPSON, S. (1973): “On the applicability of root transformations”, Linguistic Inquiry 4, pp. 46597.

Huddleston, R.; Pullum, G. (2002): The Cambridge Grammar of the English Language. Cambridge: Cambridge University Press. https://doi.org/10.1017/9781316423530

JIMÉNEZ-FERNÁNDEZ, Á. L. (2018): "Negative Preposing. Intervention and parametric variation in complement clauses", Atlantis 40(1), pp. 11-37. https://doi.org/10.28914/Atlantis-2018-40.1.01

JimÉNEZ-FERnÁNDEZ, Á. L. (2020): Syntax-Information Structure Interactions in the Sentential, Verbal and Nominal Peripheries. Newcastle: Cambridge Scholars Publishing.

JIMÉNEZ-FERNÁNDEZ, Á. L.; MIYAGAWA, s. (2014): “A feature-inheritance approach to root phenomena and parametric variation", Lingua 145, pp. 276-302. https://doi.org/10.1016/j.lingua.2014.04.008

LASNIK, H. (2003): Minimalist Investigations in Linguistic Theory. London: Routledge.

LEONETTI, M.; EsCAndelL, V. (2009): “Fronting and verum focus in Spanish”, en A. Dufter y D. Jacob (eds.): Focus and Background in Romance Languages. Amsterdam y Philadelphia, PA: John Benjamins, pp. 155-204. https://doi.org/10.1075/slcs.112.07leo

LEONETTI, M.; EsCANDELL, V. (2017): “El foco es relativo”, en Á. Gallego, Y. Rodríguez y J. Sánchez-Fernández (eds.): Relaciones Sintácticas: Homenaje a José M. Brucart y M. Lluïsa Hernanz. Barcelona: Universitat Autònoma de Barcelona, pp. 435-446.

LóPEZ, L. (2009): A Derivational Syntax for Information Structure. Oxford: Oxford University Press. https://doi.org/10.1093/acprof:oso/9780199557400.001.0001

Meinunger, A. (2004): "Verb position, verbal mood and the anchoring (potential) of sentences", en H. Lohnstein y S. Trissler (eds.): The Syntax and Semantics of the Left Periphery. Berlin: Mouton de Gruyter, pp. 313-341.

MiyagaWA, S. (2010): Why Agree? Why Move? Unifying Agreement-Based and Discourse Configurational Languages. Cambridge, MA: MIT Press. https://doi.org/10.7551/mitpress/8116.001.0001

Miyagawa, S. (2017): Agreement Beyond Phi. Cambridge, MA: MIT Press. https://doi.org/10.7551/mitpress/10958.001.0001

OJEA, A. (2016): “Categorías mixtas truncadas: La nominalización defectiva en las cláusulas relativas existenciales modales”, RLA: Revista de Lingüística Teórica y Aplicada 54(1), pp.129-147. https://doi.org/10.4067/S0718-48832016000100007 
OJEA, A. (2017): “Core intentional features in the syntactic computation: Deriving the position of the subject in Spanish", Lingua 195, pp. 72-91. https://doi.org/10.1016/j.lingua.2017.06.007

OJEA, A. (2019): “EPP satisfaction on discourse grounds: The case of locative inversion”, Syntax 22(2/3), pp. 248-273. https://doi.org/10.1111/synt.12181

ORTEGa-SAntos, I. (2020): "Dialect distance and data assessment in Chilean, Venezuelan and Puerto Rican Spanish”, manuscrito sin publicar, Universidad de Memphis, EE. UU.

PoTTS, C. (2007): “Into the conventional-implicature dimension", Philosophy Compass 2(4), pp. 665-679. https://doi.org/10.1111/j.1747-9991.2007.00089.x

QUIRK, R. S.; GREENBAUM, S; LEECH, G.; SVARTVIK, J. (1985): A Comprehensive Grammar of the English Language. London: Longman.

RADFORD, A. (2009): Analysing English Sentences: A Minimalist Approach. Cambridge: Cambridge University Press. https://doi.org/10.1017/CB09780511801617

RADFord, A. (2019): Relative Clauses: Structure and Variation in Everyday English. Cambridge: Cambridge University Press. https://doi.org/10.1017/9781108687744

RICHARDS, N. W. (1999): “Cyclicity and the ordering of multiple specifiers”, en S. Epstein y N. Hornstein (eds.): Working Minimalism. Cambridge, MA: MIT Press, pp. 127-158.

RIzZI, L. (1990): Relativized Minimality. Cambridge, MA: MIT Press.

RizzI, L. (1997): “The fine structure of the left periphery”, en L. Haegeman (ed.): Elements of Grammar: Handbook in Generative Syntax. Dordrecht: Kluwer, pp. 281-337. https://doi.org/10.1007/978-94$\underline{011-5420-87}$

RizzI, L. (2004): "Locality and left periphery", en A. Belletti (ed.): Structures and Beyond. The Cartography of Syntactic Structures. Oxford: Oxford University Press, vol. 3, pp. 223-251.

Rizzi, L. (2006): “On the form of chains: Criterial positions and ECP effects”, en L. Cheng y N. Corver (eds.): Wh-Movement: Moving on. Cambridge, MA: MIT Press, pp. 97-134.

SPEAS, M. (2000). "Evidentiality, logophoricity and the syntactic representation of pragmatic features", Lingua 14, pp. 255-276. https://doi.org/10.1016/S0024-3841(03)00030-5

SPEAS, M.; TenNy, C. (2003): “Configurational properties of point of view roles”, en A. M. Di Sciullo (ed.): Asymmetry in Grammar. Amsterdam/Philadelphia: John Benjamins, pp. 315-344. https://doi.org/10.1075/la.57.15spe

Sprouse, J.; AlmeidA, D. (2012): "Assessing the reliability of textbook data in syntax: Adger's Core Syntax", Journal of Linguistics 48(3), pp. 609-652. https://doi.org/10.1017/S0022226712000011

SPROUSE, J.; SCHÜTZE, C.; ALMEIDA, D. (2013): “A comparison of informal and formal acceptability judgments using a random sample from Linguistic Inquiry 2001-2010", Lingua 134, pp. 219-248. https://doi.org/10.1016/j.lingua.2013.07.002

SUÑER, M. (1992): "V-movement and the licensing of argumental wh-phrases in Spanish", Natural Language and Linguistic Theory 12, pp. 335-372. https://doi.org/10.1007/BF00993148

Villa-García, J. (2015): The Syntax of Multiple-Que Sentences in Spanish: Along the Left Periphery. Amsterdam/Philadelphia: John Benjamins. https://doi.org/10.1075/ihll.2

VILLATA, S.; RIZZI, L.; FRANCK, J. (2016): “Intervention effects and Relativized Minimality: New experimental evidence from graded judgments", Lingua 179, pp. 76-96. https://doi.org/10.1016/j.lingua.2016.03.004 
ZubizarretA, M. L. (1998): Word Order, Prosody and Focus. Cambridge, MA: MIT Press.

\section{Notas}

1 Este trabajo ha sido financiado por el proyecto de investigación Estructura Informativa en su Interfaz SintácticoSemántica: Comparación de Lenguas Románicas y Germánicas (PGC2018-093774-B-I00) del Ministerio de Ciencia, Innovación y Universidades de España. Versiones anteriores de este trabajo han sido presentadas en el I Encuentro de Lingüística Formal celebrado en el Colegio de México en septiembre de 2020, así como en el 43.er Congreso de AEDEAN, panel de Sintaxis, que tuvo lugar en la Universidad de Alicante en noviembre de 2019. Agradezco los comentarios de los asistentes a ambos eventos.

2 En trabajos previos (Jiménez-Fernández y Miyagawa 2014, Jiménez-Fernández 2020) se ha demostrado que, frente a la anteposición de tópico en inglés, la dislocación de tópico en español con doblado de clítico no es un FOM y, por consiguiente, es legítima en contextos subordinados que expresen presuposición. De ahí que en la traducción española en (5) sea posible anteponer el objeto cada parte siempre que se doble con el pronombre átono la. Para el mismo efecto en otras lenguas románicas, véase Haegeman (2012) y Bianchi y Frascarelli (2010).

$3 \mathrm{El}$ foco informativo es aquella categoría discursiva que recae sobre el constituyente que expresa mera información nueva (Zubizarreta 1998). En la oración (i), como respuesta a la pregunta ¿Quién se ha comido todo el pastel?, el sujeto posverbal Luis desempeña la función discursiva de foco informativo:

(i) El pastel se lo ha comido Luis.

Por otro lado, el foco informativo se describe como un FOM porque solo puede tener esta lectura informativa en contextos matrices o cuasimatrices (Leonetti y Escandell 2017).

4 La naturaleza de foco de la ANeg en inglés está ampliamente aceptada en la bibliografía sobre el tema. Así, Emonds (2004) o Haegeman (2012) indican que se trata de un tipo de foco contrastivo, pues enfatizan un contraste entre dos miembros de un conjunto (uno negativo y otro positivo). Sin embargo, esta es una cuestión controvertida en la bibliografía especializada del español. Por un lado, autores como Gallego (2007) y Batllori y Hernanz (2014) sugieren que se trata de un tipo de anteposición con rasgos de foco. Por otro lado, Leonetti y Escandell (2009) arguyen que se trata de un tipo de foco de polaridad que afecta a toda la oración y no solo al elemento antepuesto. En este trabajo, no voy a discutir si es un tipo u otro de foco (véase Jiménez-Fernández 2018 para una discusión completa sobre este particular). Lo que me interesa de la ANeg de cara a la hipótesis de este trabajo es que implica un movimiento a la izquierda causado por rasgos de foco que se puede ver bloqueado o no dependiendo del contexto sintáctico y de la lengua concreta en que se da.

5 En este trabajo me centro en los dos tipos de relativas que ya he señalado. Hay otros tipos de relativas, como pueden ser las relativas existenciales modales de (i) (Ojea 2016), las especificativas indefinidas como la de (ii) (Hooper y Thompson 1973), o las relativas denominadas kind-defining o definitorias de la clase, (Benincà 2012, Benincà y Cinque 2014) como la de (iii) (Radford 2019):

(i) Estoy buscando con quien hablar.

(ii) I saw a dress which under no circumstances would I have bought.

'Vi un vestido que bajo ningún concepto me hubiera comprado'.

(iii) He's a guy who/that gets into a lot of fights.

'Es un chico que se mete en un montón de peleas'.

La nota común de estos tres tipos es que el antecedente es siempre indefinido (o, al menos, tiene una lectura como indefinido), y por tanto la relativa (junto con el antecedente) expresa información nueva que no se puede presuponer. Como aserciones, estas relativas son en principio compatibles con FOM como la ANeg:

(iv) Syntax is a subject which only very rarely will students enjoy. (Adaptada de Radford 2009: 330, ej. 8b)

'La sintaxis es una asignatura de la que muy rara vez disfrutan los estudiantes'.

Tanto en el experimento como en el resto del artículo, no he tenido en cuenta estos tipos de relativas. Sirva esta breve nota para indicar una línea de investigación que retomaré en futuros trabajos. 
6 Otros FOM como la Inversión Locativa muestran el mismo comportamiento restringido en las relativas explicativas y especificativas, como ilustro en (i-ii) para el inglés:

(i) The rotunda, in which stands a statue of Washington, will be repainted.

(ii) *The rotunda in which stands a statue of Washington will be repainted.

'La rotonda (,) en la que se erigía la estatua de Washington (,) será pintada de nuevo'.

(Hooper y Thompson 1973: 487, ejs. 200-201)

Para un análisis reciente de estas construcciones basada en la estructura informativa y su naturaleza de FOM, véase Ojea (2019), quien ofrece una visión comparada inglés/español de este fenómeno discursivo.

7 Sobre el carácter de FOM de la Topicalización en español, basándose en los diferentes tipos de tópico propuestos por Bianchi y Frascarelli (2010), véase Jiménez-Fernández y Miyagawa (2014) y Jiménez-Fernández (2020).

8 Para diferentes implementaciones de la estructura sintáctica dentro del análisis cartográfico, véase Haegeman (2012), Villa-García (2015), Frascarelli y Jiménez-Fernández (2017, 2019), entre muchos otros. 